\title{
Homomorphisms and boundedness in topological groups
}

\author{
Roman Ger and Maciej Sablik \\ Dedicated to Professor János Aczél on his 85th birthday
}

\begin{abstract}
We consider homomorphisms from a normed space into a topological group. Assuming their boundedness in a neighbourhood (in relative topology) of an extremal point of the unit sphere, we derive their linearity (whenever it makes sense), closedness of the graph or continuity.
\end{abstract}

Mathematics Subject Classification (2010). 39B52, 39B62.

Keywords. Functional equation, bounded homomorphism, closed graph, extremal points.

\section{Introduction}

One of the typical questions asked in connection with the Cauchy functional equation is whether its solution (i.e. additive function or homomorphism) which is bounded on a set has to be continuous. This is generally true if the set is open, and holds for a larger class of convex functions in the case where the range is the real line, let us quote e.g. [2] or [9]. Kuczma's book [9] is also a good source of references for answering the forementioned question if the set has empty interior, or is thin in a sense. Usually, when the answer is positive, some kind of Steinhaus type theorem is used, guaranteeing that the algebraic sum of two sets contains a nonempty open subset. This is the case for instance when one would like to infer continuity from boundedness on the graph of a function (cf. [3,4,6,8], as well as [9]).

An interesting point in investigations concerning the Cauchy equation or its generalized form

$$
f(x+y)=F(f(x), f(y))
$$

where $F$ is a continuous binary operation in some topological space $Y$ and the domain of the unknown function $f$ is a normed space $X$, was to derive the continuity of $f$ from the continuity or merely boundedness of $f \mid V, V$ being an open subset of a sphere. Let us quote here the paper [12], where $Y$ is also a 
normed space, $F$ is addition in $Y$ and $V$ is not contained in any two parallel hyperspaces. In the case where $X$ is a real inner product space, the result "continuity on a sphere implies continuity" was proved in [11]. Baron in [1] has showed that if $X$ is a Banach space, then it is sufficient to assume Borel measurability of $f$ and $F$ to get the continuity of solutions of $(*)$.

Kucia in [7] pointed out that continuity at an extremal point of a sphere is essential in such considerations. Among others she has proved that the continuity of $f \mid S$, where $S$ stands for the unit sphere in a normed space with $\operatorname{dim} X \geq 2$ at an extremal point of $S$ implies its continuity, provided $F(f(X) \times f(X))$ is a topological group. P. Volkmann in a non-published remark observed that this result follows from [12] if $Y$ is a real normed space.

In the present paper we are concerned with boundedness on a neighbourhood of an extremal point of a sphere. While some of our results can be derived from the earlier ones, we give independent proofs and obtain analogons for Jensen convex functions. Also, we show that a weaker kind of boundedness implies convexity or linearity which is nothing else but the continuity of an additive mapping with respect to the algebraic topology. Since obviously $(f(X), F)$ (cf. $(*)$ ) is an abelian group with $f(0)$ as neutral element, in Sect. 3 we deal with homomorphisms from a normed space into a topological group, and get that "boundedness on a neighbourhood of an extremal point of a sphere implies continuity" for a large class of topological groups $Y$.

\section{Real valued homomorphisms}

Throughout the whole paper we assume that $(X,\|\cdot\|)$ is a real normed space. For any $x \in X$ and $r>0$ we denote by $B(x, r)$ the closed ball with radius $r$, centered at $x$, and by $S(x, r)$ the corresponding sphere. $S$ will stand for the unit sphere in $X$, i.e.

$$
S:=\{x \in X:\|x\|=1\} .
$$

If $e \in S$ and $\varepsilon>0$ then we put

$$
S_{e, \varepsilon}:=S \cap B(e, \varepsilon) .
$$

Obviously, $S_{e, \varepsilon}$ is a neighbourhood of $e$ on $S$, in the topology inherited from $X$. Let us recall that $e$ belonging to a subset $A$ of $X$ is an extremal point of $A$ iff it is not an internal point of any segment contained in $A$. For a function $f: U \rightarrow V$ by $\operatorname{gr}(f)$ we denote the graph of $f$, i.e. $\{(u, v) \in U \times V: v=f(u)\}$.

We start with the following technical lemma.

Lemma 2.1. Let $X$ be a two-dimensional normed space with norm $\|\cdot\|$. Assume that $e, x_{0} \in S$ are linearly independent. Then there is a concave function $s:(-1,1) \longrightarrow \mathbb{R}$ such that

$$
S \cap\left((0, \infty) e+(-1,1) x_{0}\right)=\left\{t x_{0}+s(t) e: t \in(-1,1)\right\} .
$$


In other words the set $S \cap\left\{s e+t x_{0}: s \in(0, \infty), t \in(-1,1)\right\}$ is the graph of a concave function (in the coordinate system given by $x_{0}$ and $e$ ).

Proof. Put $V:=\left\{s e+t x_{0}: s \in(0, \infty),|t|<1\right\}$. Obviously, the set $V$ is open and $e \in V$. Hence $V \cap S$ is a neighbourhood of $e$ in the relative topology on $S$. We show that for every $t \in(-1,1)$ the line

$$
t x_{0}+\mathbb{R} e
$$

intersects $V \cap S$ in exactly one point.

Indeed, fix a $t \in(-1,1)$ and consider the function $\varphi_{t}: \mathbb{R} \rightarrow \mathbb{R}$ given by

$$
\varphi_{t}(s)=\left\|s e+t x_{0}\right\| .
$$

The function $\varphi_{t}$ is convex and

$$
\varphi_{t}(0)=|t|<1 .
$$

On the other hand we have for every $s \in \mathbb{R}$

$$
\varphi_{t}(s) \geq\|s e\|-\left\|t x_{0}\right\|=|s|-|t|
$$

whence $\lim _{s \rightarrow \infty} \varphi_{t}(s)=\infty$. This together with (1) and the convexity of $\varphi_{t}$ implies that there exists exactly one $s=s(t)>0$ such that

$$
\varphi_{t}(s(t))=1 \text {. }
$$

Indeed, $\varphi_{t} \mid[0, \infty)$ as a convex function satisfying (1) and tending to $\infty$ at $\infty$, is either strictly increasing, or decreases to a minimum (less than 1) attained at a point $\sigma>0$ and then strictly increases. In particular, $\varphi_{t} \mid[\sigma, \infty)$ is strictly increasing for some $\sigma \in[0, s(t))$.

We have defined the function $s:(-1,1) \rightarrow(0, \infty)$ so that

$$
V \cap S=\left\{s(t) e+t x_{0}: t \in(-1,1)\right\} .
$$

It follows that $V \cap S$ is the graph of the function $s$ with respect to the system of coordinates induced by $e$ and $x_{0}$. Let us show now that the function $s$ is concave.

Fix $t, t^{\prime} \in(-1,1)$ and $\lambda \in(0,1)$ and denote by $\beta$ the number $\lambda t+(1-\lambda) t^{\prime} \in$ $(-1,1)$. Now, because $s(t) e+t x_{0}, s\left(t^{\prime}\right) e+t^{\prime} x_{0} \in S$ we have

$$
\begin{aligned}
1 & =\lambda+(1-\lambda)=\left\|\lambda s(t) e+\lambda t x_{0}\right\|+\left\|(1-\lambda) s\left(t^{\prime}\right) e+(1-\lambda) t^{\prime} x_{0}\right\| \\
& \geq\left\|\left(\lambda s(t)+(1-\lambda) s\left(t^{\prime}\right)\right) e+\left(\lambda t+(1-\lambda) t^{\prime}\right) x_{0}\right\| \\
& =\left\|\left(\lambda s(t)+(1-\lambda) s\left(t^{\prime}\right)\right) e+\beta x_{0}\right\| .
\end{aligned}
$$

Since $\varphi_{\beta} \mid[\sigma, \infty)$ is increasing for some $\sigma \in[0, s(\beta))$ and $\varphi_{\beta}(0)<1$, the above inequality implies

$$
s\left(\lambda t+(1-\lambda) t^{\prime}\right) \geq \lambda s(t)+(1-\lambda) s\left(t^{\prime}\right)
$$

thus proving the concavity of $s$ and concluding the proof. 
Remark 2.2. The concavity of the function $s$ whose graph is $V \cap S$ is obviously due to the convexity of the closed unit ball.

Remark 2.3. If we admit additionally in Lemma 2.1 that $e$ is an extremal point of $S$, and take an arbitrary $\delta \in(0,1)$ then the restriction of the function $s$ defined in the proof to the interval $(-\delta, \delta)$ is not affine. Indeed, suppose that $s \mid(-\delta, \delta)$ is affine, fix a $t \in(-\delta, \delta) \backslash\{0\}$ and set $x_{1}=s(t) e+t x_{0}$ and $x_{2}=s(-t) e-t x_{0}$. Then $x_{1}, x_{2} \in V \cap S \backslash\{e\}$ and since $s(0)=1$ we get

$$
\frac{x_{1}+x_{2}}{2}=\frac{s(t)+s(-t)}{2} e=s(0) e=e .
$$

Thus the point $e$ cannot be extremal.

The following lemma is a Steinhaus type theorem for thin sets in $\mathbb{R}^{2}$.

Lemma 2.4. Under the assumptions of Lemma 2.1, if e is an extremal point of $S$ then for every $\varepsilon>0$

$$
\operatorname{int}\left(S_{e, \varepsilon}+S_{e, \varepsilon}\right) \neq \emptyset \text {. }
$$

Proof. We keep the notation from Lemma 2.1. Obviously, it is enough to prove the assertion for $\varepsilon$ small enough to have

$$
S_{e, \varepsilon} \subset V \cap S=\left\{s(t) e+t x_{0}: t \in(-1,1)\right\} .
$$

Let $\delta \in(0,1)$ be such that

$$
\left\{s(t) e+t x_{0}: t \in(-\delta, \delta)\right\} \subset S_{e, \varepsilon} .
$$

The function $s \mid(-\delta, \delta)$ is concave and hence continuous. By Remark $2.3 s$ is not affine. Now, repeating the argument from the proof of [9, Theorem 9.5.2] we infer that

$$
\operatorname{int}(\operatorname{gr}(s \mid(-\delta, \delta))+\operatorname{gr}(s \mid(-\delta, \delta))) \neq \emptyset
$$

where $\operatorname{gr}(s \mid(-\delta, \delta))$ denotes the graph of the corresponding function in the system of coordinates generated by $x_{0}$ and $e$. This yields obviously the assertion of the lemma.

Let us extend the previous result to the case of an arbitrary two-dimensional normed space. We have the following.

Lemma 2.5. Let $\operatorname{dim} X=2$ and assume that $e$ is an extremal point of $S$. Then for every $\varepsilon>0$

$$
\operatorname{int}\left(S_{e, \varepsilon}+S_{e, \varepsilon}\right) \neq \emptyset \text {. }
$$

Proof. Let $\{u, v\}$ be an algebraic base of $X$. Define a mapping $h: X \rightarrow \mathbb{R}^{2}$ by

$$
h(\alpha u+\beta v):=(\alpha, \beta),
$$

and let the functional $\|\cdot\|_{h}: \mathbb{R}^{2} \rightarrow \mathbb{R}$ be given by

$$
\|(\alpha, \beta)\|_{h}:=\|\alpha u+\beta v\| \text {. }
$$


It is easy to check that $\|\cdot\|_{h}$ is a norm in $\mathbb{R}^{2}$ and $h$ is an isometric isomorphism between $(X,\|\cdot\|)$ and $\left(\mathbb{R}^{2},\|\cdot\|_{h}\right)$. This implies in particular that $h(e)$ is an extremal point of the unit sphere in $\left(\mathbb{R}^{2},\|\cdot\|_{h}\right)$, i.e. of the set $S_{h}:=\left\{(\alpha, \beta) \in \mathbb{R}^{2}:\|(\alpha, \beta)\|_{h}=1\right\}$. We also have

$$
h\left(S_{e, \varepsilon}\right)=h(B(e, \varepsilon) \cap S)=h(B(e, \varepsilon)) \cap h(S)=B(h(e), \varepsilon) \cap S_{h}
$$

and thus $h\left(S_{e, \varepsilon}\right)$ is a neighbourhood of $h(e)$ on the sphere $S_{h}$. Hence, in view of Lemma 2.4 and linearity of $h$ we get

$$
\operatorname{int}_{\mathbb{R}^{2}}\left(h\left(S_{e, \varepsilon}\right)+h\left(S_{e, \varepsilon}\right)\right)=\operatorname{int}_{\mathbb{R}^{2}} h\left(S_{e, \varepsilon}+S_{e, \varepsilon}\right) \neq \emptyset .
$$

Since $h$ is a homeomorphism we infer that $\operatorname{int}_{X}\left(S_{e, \varepsilon}+S_{e, \varepsilon}\right) \neq \emptyset$ which ends the proof.

Now we pass to the study of additive functionals bounded on thin sets. Their regularity will be a consequence of slightly more general results stated for Jensen convex functions. Our first result concerns convex functions bounded on an open subset of $S$.

Lemma 2.6. Let $(X,\|\cdot\|)$ be a real normed space, let $D \supset S$ be an open and convex subset and assume that $\operatorname{dim} X \geq 2$. If $a: D \rightarrow \mathbb{R}$ is convex and bounded from above on a nonempty subset of $S$ which is open in $S$ then a is continuous.

Proof. Without loss of generality we may assume that $\varepsilon \in(0,1)$ and $e \in S$ are such that

$$
M:=\sup \left\{a(z): z \in S_{e, \varepsilon}\right\}<\infty .
$$

Consider the cone

$$
C:=\bigcup_{\lambda \in[0,1]} \lambda S_{e, \varepsilon} .
$$

If $x \in C$ then $x=\lambda z$ for some $\lambda \in[0,1]$ and $z \in S_{e, \varepsilon}$. Since $a$ is convex we get in view of $(2)$

$$
a(x) \leq \lambda a(z)+(1-\lambda) a(0) \leq M+a(0),
$$

which means that $a$ is bounded from above in $C$. To conclude the proof it is enough to show that int $C \neq \emptyset$ and use well known results on the continuity of convex functions bounded from above (cf. $[8,9]$ or $[10]$ ). We will show that actually

$$
B\left(e, \frac{1}{2} \varepsilon\right) \cap B(0,1) \subset C .
$$

Let us note that $\left(1-\frac{1}{4} \varepsilon\right) e \in B\left(e, \frac{1}{2} \varepsilon\right) \cap B(0,1)$ and so the intersection on the lefthand side is a nonempty open set. 
To prove (3) let $x \in B\left(e, \frac{1}{2} \varepsilon\right) \cap B(0,1)$. Then $0<\|x\|<1$ and $\|x-e\|<\frac{1}{2} \varepsilon$ and therefore also

$$
1-\|x\|=\|e\|-\|x\| \leq\|e-x\|<\frac{1}{2} \varepsilon .
$$

Taking the above inequality into account we get

$$
\begin{aligned}
\left\|\frac{x}{\|x\|}-e\right\| & \leq\left\|\frac{x}{\|x\|}-x\right\|+\|x-e\|=\left(\frac{1}{\|x\|}-1\right)\|x\|+\|x-e\| \\
& =(1-\|x\|)+\|x-e\|<\frac{1}{2} \varepsilon+\frac{1}{2} \varepsilon=\varepsilon,
\end{aligned}
$$

which yields $\frac{x}{\|x\|} \in S_{e, \varepsilon}$ because of course $\frac{x}{\|x\|} \in S$. As a consequence we obtain

$$
x=\|x\| \frac{x}{\|x\|} \in C,
$$

which ends the proof.

We will also need the following characterization of convex functionals.

Lemma 2.7. Let $X$ and $D$ be as in the previous lemma, and let $e \in S$. A Jensen convex functional $a: D \rightarrow \mathbb{R}$ is convex if and only if for any two-dimensional space $X_{0} \subset X$ containing e the restriction $a \mid X_{0} \cap D$ is convex.

Proof. The necessity is obvious, and to prove sufficiency let us fix arbitrarily $x, y \in D$. If $x$ and $y$ are linearly dependent then they both belong to a twodimensional space $X_{0}$ containing $e$ and therefore

$$
a(\lambda x+(1-\lambda) y) \leq \lambda a(x)+(1-\lambda) a(y)
$$

for every $\lambda \in[0,1]$. Suppose that $x$ and $y$ are linearly independent. Let $\delta>0$ be such that for every $s, t \in(-\delta, \delta)$ we have $s x+t y, s x+e, t y-e \in D$. We may assume that $\delta$ is small enough so that $a$ is bounded from above on $(-\delta, \delta) x+e$ and $(-\delta, \delta) y-e$. This is because restrictions of $a$ to the plane containing $x$ and $e$ as well as to the one containing $y$ and $e$ are convex and hence continuous with respect to relative topologies. From the Jensen convexity of $a$ it follows that

$$
a\left(\frac{s x+t y}{2}\right) \leq \frac{a(s x+e)+a(t y-e)}{2}
$$

which means that $a$ is bounded from above on the set $\left\{\frac{s x+t y}{2}: s, t \in(-\delta, \delta)\right\}$ which is open in the plane spanned by $x$ and $y$. Hence in view of the BernsteinDoetsch Theorem (cf. $[2,9]$ ) the continuity of $a$ follows, which implies that (4) holds for every $\lambda \in[0,1]$, and the proof is completed.

Our main result concerning Jensen convex functionals is presented in the following Proposition. Let us note that in the case where $X=\mathbb{R}^{2}$ the statement can be derived from [11, Theorem 7]. 
Proposition 2.8. Let $(X,\|\cdot\|)$ be a real normed space with $\operatorname{dim} X \geq 2$. Let $D \subset X$ be a convex and open set containing $S$, and let $e$ be an extremal point of $S$. A Jensen convex functional $a: D \rightarrow \mathbb{R}$ is convex if and only if for every two-dimensional plane $X_{0} \subset X$ containing e there exists $\varepsilon>0$ such that $a \mid X_{0} \cap S_{e, \varepsilon}$ is bounded from above.

Proof. Let $x \in X$ be a vector linearly independent of $e$ and set

$$
X_{0}:=\{\alpha x+\beta e: \alpha, \beta \in \mathbb{R}\} .
$$

Obviously, $e$ is an extremal point of the unit sphere $S_{X_{0}}=S \cap X_{0}$ in $X_{0}$. Choose $\varepsilon>0$ so that the set $S_{X_{0}, e, \varepsilon}=X_{0} \cap S_{e, \varepsilon}$ is a neigbourhood of $e$ on the sphere $S_{X_{0}}$ on which $a$ is bounded from above. By Jensen convexity, $a$ is bounded from above also on $\frac{1}{2}\left(S_{X_{0}, e, \varepsilon}+S_{X_{0}, e, \varepsilon}\right)$, and hence, in view of Lemma 2.5, on an open subset of $X_{0} \cap D$. Due to the Bernstein-Doetsch Theorem $a \mid X_{0} \cap D$ is continuous (with respect to the topology of $X_{0}$ ), and hence convex in $X_{0} \cap D$. Since $x$ was chosen arbitrarily we can apply Lemma 2.7 to get our assertion.

We get the following corollary for additive functionals.

Corollary 2.9. Let $(X,\|\cdot\|)$ be a real normed space and assume that $\operatorname{dim} X \geq 2$. Let $e$ be an extremal point of the unit sphere $S \subset X$. An additive functional $a: X \rightarrow \mathbb{R}$ is linear if and only if for every two-dimensional subspace $X_{0} \subset X$ containing e there exists $\varepsilon>0$ such that $a \mid X_{0} \cap S_{e, \varepsilon}$ is bounded above or below.

Proof. It is enough to show that $a$ is homogeneous. Let $x \in X$ be a vector linearly independent of $e$ and set

$$
X_{0}:=\{\alpha x+\beta e: \alpha, \beta \in \mathbb{R}\} .
$$

Obviously, $e$ is an extremal point of the unit sphere $S_{X_{0}}=S \cap X_{0}$ in $X_{0}$. Replacing $a$ by $-a$, if necessary, we choose an $\varepsilon>0$ so that $a \mid X_{0} \cap S_{e, \varepsilon}$ is bounded from above. Applying Proposition 2.7 in the case $X=X_{0}$ we see that $a \mid X_{0}$ is continuous (with respect to the topology of $X_{0}$ ), and hence linear, i.e.

$$
a(\alpha x+\beta e)=\alpha a(x)+\beta a(e)
$$

for every $\alpha, \beta \in \mathbb{R}$. Putting $\beta=0$ in the above formula we get

$$
a(\alpha x)=\alpha a(x)
$$

for every $\alpha \in \mathbb{R}$, and for any $x$ linearly independent of $e$. To get homogeneity for vectors parallel to $e$, let us fix a $\lambda \in \mathbb{R}$ and put $\alpha=0$ in (5). Then for every $\beta \in \mathbb{R}$ we have

$$
a(\beta(\lambda e))=a((\beta \lambda) e)=\beta \lambda a(e)=\beta a(\lambda e),
$$

which concludes the proof. 
The assumption we made in the above Corollary means that $a$ is bounded on some segments of great circles passing through the extremal point $e$, allowing the segment and the kind of boundedness depend on the circle. The reader may think that this kind of assumption is motivated mainly by the technique of the proof. However, as it follows from our final result in this section, the condition appearing in Proposition 2.7 is the best we can expect from a linear discontinuous functional. Boundedness on a spherical neighbourhood of $e$ simply means that the functional is continuous. Indeed, we have the following.

Theorem 2.10. Let $(X,\|\cdot\|)$ be a real normed space and assume that $\operatorname{dim} X \geq 2$. Let $e$ be an extremal point of the unit sphere $S \subset X$. An additive functional $a: X \rightarrow \mathbb{R}$ is continuous (i.e. $a \in X^{*}$ ) if and only if it is bounded above or below on $S_{e, \varepsilon}$ for some $\varepsilon>0$.

Proof. In view of Corollary 2.9, $a$ is linear, and in particular convex. Thus, the assertion follows from Lemma 2.6.

Remark 2.11. The above result follows from [12]. However, we have given an independent proof here since our Theorem 2.10 is in fact a corollary from a more general Jensen convex case.

In the present section we have inferred linearity or continuity (resp. convexity or continuity) of additive (resp. Jensen convex) mappings from their boundedness on some spherical neighbourhoods of extremal points. Although the results are formulated for the case of the unit sphere, they are easily applicable in a general case. Suppose for instance that $D$ is an open and convex subset of $X$ and $a: D \rightarrow \mathbb{R}$ is Jensen convex. Further, let $B\left(x_{0}, r\right)$ be a closed ball contained in $\mathrm{D}$, and suppose that $e^{\prime}$ is an extremal point of $S^{\prime}=\left\{x \in X:\left\|x-x_{0}\right\|=r\right\}$. Define $\tau: X \rightarrow X$ by $\tau(x)=r x+x_{0}$. Then $a \circ \tau$ is a Jensen convex mapping defined on $\tau^{-1}(D)$ which is a convex and open neighbourhood of 0 . Moreover, $\tau^{-1}\left(B\left(x_{0}, r\right)\right)$ is the unit ball and $e=\tau^{-1}\left(e^{\prime}\right)$ is its extremal point. Also, $a$ is bounded on a set $V \subset D$ iff $a \circ \tau$ is bounded on $\tau^{-1}(V)$. Let us note that $V$ is open in $S^{\prime}$ if and only if $\tau^{-1}(V)$ is open in $S$. Moreover, $a$ is convex (continuous) iff so is $a \circ \tau$. Taking this into account one can easily derive the following generalized versions of Proposition 2.7 and Lemma 2.6.

Proposition 2.12. Let $(X,\|\cdot\|)$ be a real normed space with $\operatorname{dim} X \geq 2$. Let $D \subset X$ be a convex and open set. Further, let e be an extremal point of a sphere $S\left(x_{0}, r\right)$ contained in $D$. A Jensen convex functional $a: D \rightarrow \mathbb{R}$ is convex if and only if for every two-dimensional plane $X_{0} \subset X$ containing $e$ and $x_{0}$ the function $a \mid X_{0} \cap S$ is bounded above on a neighbourhood of $e$. 
Lemma 2.13. Let $(X,\|\cdot\|)$ be a real normed space with $\operatorname{dim} X \geq 2$, and $D$ an open and convex subset of $X$. If $a: D \rightarrow \mathbb{R}$ is convex and bounded above on a nonempty and relatively open subset of a sphere contained in $D$ then $a$ is continuous.

Similarly, one can get the following generalizations of Corollary 2.9 and Theorem 2.10.

Corollary 2.14. Let $(X,\|\cdot\|)$ be a real normed space and assume that $\operatorname{dim} X \geq 2$. Let $e$ be an extremal point of a sphere $S\left(x_{0}, r\right)$. An additive functional $a: X \rightarrow$ $\mathbb{R}$ is linear if and only if for every two-dimensional subspace $X_{0} \subset X$ containing $e$ and $x_{0}$ the function $a \mid X_{0} \cap S\left(x_{0}, r\right)$ is bounded above or below on a (relative) neighbourhood of $e$.

Theorem 2.15. Let $(X,\|\cdot\|)$ be a real normed space and assume that $\operatorname{dim} X \geq 2$. Let $e$ be an extremal point of a sphere $S\left(x_{0}, r\right)$. An additive functional $a: X \rightarrow$ $\mathbb{R}$ is continuous (i.e. $a \in X^{*}$ ) if and only if it is bounded above or below on a (relative) spherical neighbourhood of e.

\section{General case}

In the present section we extend the results obtained earlier to the case of mappings taking values in some topological groups. Let us recall that a mapping $a$ defined in a topological group $H$ and taking values in a topological group $G$ is called weakly continuous if $\chi \circ a$ is continuous for every continuous real character $\chi$ of the group $G$. By $\widehat{G}_{r}$ we denote the group of all continuous real characters of the group $G$.

We start with the following closed graph result.

Lemma 3.1. Let $H$ and $G$ be topological groups and assume that $\widehat{G}_{r}$ separates points of $G$. If $a: H \rightarrow G$ is additive and weakly continuous then $\operatorname{gr}(a)$ is closed.

Proof. Let $\left(x_{0}, y_{0}\right) \in(H \times G) \backslash \operatorname{gr}(a)$, i.e. $y_{0} \neq a\left(x_{0}\right)$. Let $\chi \in \widehat{G}_{r}$ be such that $\chi\left(y_{0}\right) \neq \chi\left(a\left(x_{0}\right)\right)$. Since $\chi \circ a$ is continuous, so is the function $\varphi: H \times G \rightarrow \mathbb{R}$ given by

$$
\varphi(x, y):=\chi(a(x))-\chi(y)
$$

(as a function of two variables). As $\varphi\left(x_{0}, y_{0}\right) \neq 0$ we infer (in view of the continuity of $\varphi$ ) that there exist a neighbourhood $U \subset H$ of $x_{0}$ and a neighbourhood $V \subset G$ such that for all $(x, y) \in U \times V$ we have

$$
\varphi(x, y) \neq 0
$$

i.e.

$$
\chi(a(x)) \neq \chi(y)
$$


for all $(x, y) \in U \times V$. In particular, we get that

$$
U \times V \subset(H \times G) \backslash \operatorname{gr}(a)
$$

which means that $\operatorname{gr}(a)$ is closed because $\left(x_{0}, y_{0}\right)$ was chosen arbitrarily.

We say that a subset $Z$ of a connected topological group $(G,+)$ is bounded iff for every neighbourhood $V$ of the neutral element of $G$ there exists a positive integer $n$ such that

$$
Z \subset \underbrace{V+\cdots+V}_{n} .
$$

Now we can formulate our next theorem.

Theorem 3.2. Let $(X,\|\cdot\|)$ be a real normed space, and let $(G,+)$ be a connected topological group such that $\widehat{G}_{r}$ separates the points of $G$. If $a: X \rightarrow G$ is an additive mapping which is bounded on a set $S_{e, \varepsilon}$ for some $\varepsilon>0$ and $e \in S$ being an extremal point of $S$ then $\operatorname{gr}(a)$ is closed in $X \times G$.

Proof. In view of Lemma 3.1 it is enough to show that $a$ is weakly continuous. Let us fix a $\chi \in \widehat{G}_{r}$ and define $f: X \rightarrow \mathbb{R}$ by $f=\chi \circ a$. Of course, $f$ is additive. Since $\chi$ is continuous there exists a neighbourhood $V$ of the neutral element in $G$ such that

$$
\chi(V) \subset(-1,1) .
$$

Let $e$ be an extremal point of $S, \varepsilon>0$ and $\mathbb{N}$ be such that

$$
a\left(S_{e, \varepsilon}\right) \subset \underbrace{V+\cdots+V}_{n} \text {. }
$$

Consequently we get

$$
\begin{aligned}
f\left(S_{e, \varepsilon}\right) & =\chi\left(a\left(S_{e, \varepsilon}\right)\right) \subset \chi(\underbrace{V+\cdots+V}_{n})=\underbrace{\chi(V)+\cdots+\chi(V)}_{n} \\
& \subset \underbrace{(-1,1)+\cdots+(-1,1)}_{n}=(-n, n),
\end{aligned}
$$

which means that

$$
|f(x)| \leq n
$$

for every $x \in S_{e, \varepsilon}$. Hence, in view of Theorem 2.10 the functional $f$ is continuous which concludes the proof.

According to [5, Corollary 24.35], if $G$ is a locally compact topological group then $\widehat{G}_{r}$ admits sufficiently many real continuous characters (i.e. $\widehat{G}_{r}$ separates points of $G$ ) if and only if the group of its characters (the dual group) is connected. Therefore we get the following. 
Corollary 3.3. Let $(X,\|\cdot\|)$ be a real normed space, and let $(G,+)$ be a connected and locally compact topological group with a connected dual group. If $a: X \rightarrow G$ is an additive mapping bounded on $S_{e, \varepsilon}$ for some $\varepsilon>0$ and some $e \in S$ being an extremal point of $S$ then $\operatorname{gr}(a)$ is closed in $X \times G$.

Another consequence of Theorem 3.2 is the following result for additive mappings with values in a real Fréchet space, i.e. in a real locally convex linear complete metric space.

Theorem 3.4. Let $(X,\|\cdot\|)$ be a real Banach space and let $G$ be a real Fréchet space. If $a: X \rightarrow G$ is an additive mapping bounded on $S_{e, \varepsilon}$ for some $\varepsilon>0$ and some $e \in S$ being an extremal point of $S$ then a is continuous.

Proof. We have $\widehat{G}_{r}=G^{*}$ in the present case, and since $G$ is locally convex, $G^{*}$ separates points of $G$. Applying now Theorem 3.2 and the closed graph principle we obtain the continuity of $a$.

Open Access. This article is distributed under the terms of the Creative Commons Attribution Noncommercial License which permits any noncommercial use, distribution, and reproduction in any medium, provided the original author(s) and source are credited.

\section{References}

[1] Baron, K.: On the continuity of additive-like functions and Jensen convex functions which are Borel on a sphere. In: Daróczy Z., Páles, Zs. (eds.) Functional EquationsResults and Advances, pp. 17-20. Kluwer Academic Publishers, Dordrecht 2002

[2] Bernstein, F., Doetsch, G.: Zur Theorie der konvexen Funktionen. Math. Ann. 76, 514$526(1915)$

[3] Ger, R.: Note on convex functions bounded on regular hypersurfaces. Demonstratio Math. 6, 97-103 (1973)

[4] Ger, R.: Thin sets and convex functions. Bull. Acad. Pol. Sci. Sèrie Des Sci. Math. Atr. Et Phys. 21, 413-416 (1973)

[5] Hewitt, E., Ross, K.E.: Abstract Harmonic Analysis I. Springer, Berlin (1979)

[6] Jabłoński, W.: On a class of sets connected with a convex function. Abh. Math. Sem. Univ. Hamburg 69, 35-43 (1999)

[7] Kucia, A.: Continuity of additive functions. (In Polish) Talk at the V Krajowe Seminarium "Wypukłe funkcje wielowartościowe". (Bystra, 17-20. 10.2001), Proceedings, pp. $11-12$

[8] Kuczma, M.: On some set classes occurring in the theory of convex functions. Comment. Math. Prace Mat. 17, 127-135 (1973)

[9] Kuczma, M.: An introduction to the theory of functional equations and inequalities. Cauchy's equation and Jensen's inequality. PWN Uniwersytet Śląski, WarszawaKraków-Katowice (see also: M. Kuczma, An introduction to the theory of functional equations and inequalities. Cauchy's Equation and Jensen's Inequality, 2nd edn. Birkhäuser Verlag 2009) (1985)

[10] Mehdi, M.R.: On convex functions. J. London Math. Soc. 39, 321-326 (1964)

[11] Ring, W., Schöpf, P., Schwaiger, J.: On functions continuous on certain classes of 'thin' sets. Publ. Math. Debrecen 51, 205-224 (1997)

[12] Volkmann, P., Walter, W.: A condition for the continuity of additive operators. Ann. Differential Equations 39, 63-66 (1987) 
Roman Ger and Maciej Sablik

Institute of Mathematics

Silesian University

Bankowa 14

40-007 Katowice

Poland

e-mail: roman.ger@us.edu.pl

e-mail: maciej.sablik@us.edu.pl

Received: January 6, 2010

Revised: May 21, 2010 